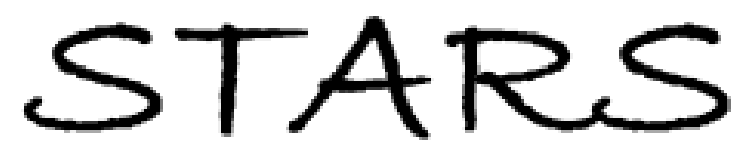

University of Central Florida

STARS

Faculty Bibliography 2000s

Faculty Bibliography

$1-1-2007$

\title{
Self-bending of dark and gray photorefractive solitons
}

M. I. Carvalho

M. Facão

D. N. Christodoulides

University of Central Florida

Find similar works at: https://stars.library.ucf.edu/facultybib2000

University of Central Florida Libraries http://library.ucf.edu

This Article is brought to you for free and open access by the Faculty Bibliography at STARS. It has been accepted for inclusion in Faculty Bibliography 2000s by an authorized administrator of STARS. For more information, please contactSTARS@ucf.edu.

\section{Recommended Citation}

Carvalho, M. I.; Facão, M.; and Christodoulides, D. N., "Self-bending of dark and gray photorefractive solitons" (2007). Faculty Bibliography 2000s. 6913.

https://stars.library.ucf.edu/facultybib2000/6913

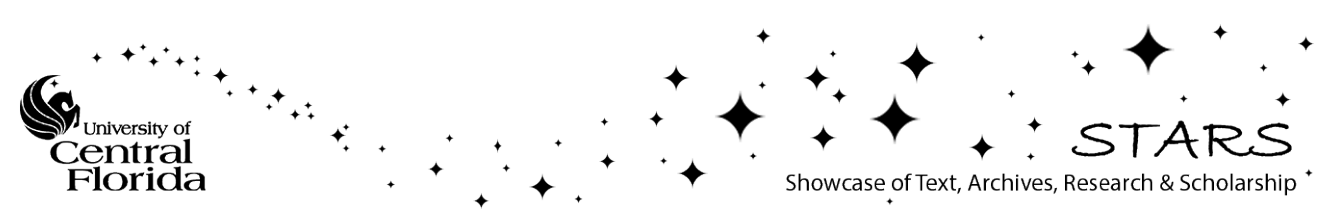




\title{
Self-bending of dark and gray photorefractive solitons
}

\author{
M. I. Carvalho, ${ }^{1}$ M. Facão, ${ }^{2}$ and D. N. Christodoulides ${ }^{3}$ \\ ${ }^{1}$ Department of Electrical Engineering and Computers, Porto University, Rua Dr. Roberto Frias, 4200-465 Porto, Portugal \\ ${ }^{2}$ Department of Physics, Aveiro University, Campus Universitário de Santiago, 3810-193 Aveiro, Portugal \\ ${ }^{3}$ School of Optics/CREOL, University of Central Florida, Orlando, Florida 32816-2700, USA \\ (Received 30 October 2006; revised manuscript received 26 March 2007; published 10 July 2007)
}

\begin{abstract}
We investigate the effects of diffusion on the evolution of steady-state dark and gray spatial solitons in biased photorefractive media. Numerical integration of the nonlinear propagation equation shows that the soliton beams experience a modification of their initial trajectory, as well as a variation of their minimum intensity. This process is further studied using perturbation analysis, which predicts that the center of the optical beam moves along a parabolic trajectory and, moreover, that its minimum intensity varies linearly with the propagation distance, either increasing or decreasing depending on the sign of the initial transverse velocity. Relevant examples are provided.
\end{abstract}

DOI: 10.1103/PhysRevE.76.016602

PACS number(s): 42.65.Tg, 42.65.Hw, 42.65.Jx

\section{INTRODUCTION}

Since their first experimental observation [1], optical spatial solitons in photorefractive media have been the focus of considerable attention. Among the reasons for the interest in these self-trapped entities, the most important are probably the possibility of observing them at $\mu \mathrm{W}$ power levels and in two transverse dimensions [2,3]. Moreover, photorefractive media host a number of interesting nonlinear phenomena, that allowed the prediction and observation of photorefractive solitons in the form of bright or dark beams [3-5], as scalar or vector [6,7], resulting from coherent or incoherent illumination [8-10] and even as discrete solitons in waveguide arrays $[11,12]$. This variety of behavior makes them excellent candidates to beam steering and beam manipulation.

Photorefractive spatial solitons are possible when the process of diffraction is exactly balanced by light-induced photorefractive waveguiding. Most often, the photorefractive nonlinearity responsible for the self-trapping of solitary beams relies on the application of an external electric field. Under strong bias conditions, drift dominates the transport mechanism. In this case, bright, dark, and gray solitary wave beams have been investigated in steady-state conditions $[4,5,13]$. However, in some situations diffusion effects cannot be neglected. Effectively, the diffusion process introduces an asymmetric tilt in the light-induced photorefractive waveguide, thus affecting the propagation characteristics of these solitons. Until now, diffusion effects in photorefractive media have been investigated solely for bright solitary beams. The results obtained show that these optical beams move along parabolic trajectories [14-17], in a distortionless and stable fashion $[18,19]$. Even though the evolution of dark and gray photorefractive solitons under diffusion effects has not yet been considered, it is important to refer that in the low amplitude regime, the photorefractive nonlinearity turns to be cubic, similar to the Kerr nonlinearity. In this limit, the model including drift and diffusion resembles the model used for nonlinear propagation of femtosecond pulses in fibers, where the effect of intrapulse Raman scattering is important. In this context, the phenomenon is called soliton self- frequency shift and the behavior of existing dark pulses was already approached by a perturbation method [20-23].

In this paper we investigate the effects of the diffusion process on the evolution of steady-state dark and gray (1 +1 ) D solitons in biased photorefractive media. Our numerical results indicate that the shape of these optical beams remains approximately invariant during propagation, although the minimum value it attains varies considerably. Moreover, we also find that the trajectory of the optical soliton is modified as a result of the diffusion transport mechanism. This process is further studied using perturbation methods which involve the modified conservation laws of the nonlinear wave equation in a moving coordinate frame. Our analysis predicts that the optical beam moves along a parabolic trajectory and that its minimum intensity varies linearly with the propagation distance, either increasing or decreasing depending on the sign of the initial transverse velocity. These analytical results are then compared to those obtained numerically and are found to be in good agreement.

\section{THEORETICAL MODEL AND DIFFUSIONLESS SOLUTIONS}

We start our analysis by considering a planar optical beam that propagates in a photorefractive material along the $z$ axis and is allowed to diffract only along the $x$ direction, which coincides with the optical $c$ axis of the crystal. Thus, we will be dealing with $(1+1) \mathrm{D}$ optical beams, and any $y$ variation has been neglected. Let us assume that the optical beam is linearly polarized along $x$ and that the external bias field is applied in the same direction. Under these conditions, the evolution of the optical beam depends on the so-called photorefractive screening nonlinearity and is governed by the equation [5]

$$
i \frac{\partial \phi}{\partial z}+\frac{1}{2 k} \frac{\partial^{2} \phi}{\partial x^{2}}-\frac{k_{0}}{2}\left(n_{e}^{3} r_{33} E_{\mathrm{sc}}\right) \phi=0,
$$

where $\phi$ is the slowly varying envelope of the optical beam, $k=k_{0} n_{e}$, with $k_{0}=2 \pi / \lambda_{0}$ being the free-space wave vector of the lightwave employed and $n_{e}$ the unperturbed extraordinary 
index of refraction, $r_{33}$ is the electro-optic coefficient and $E_{\mathrm{sc}}$ is the space-charge electric field resulting from the external bias electric field and the redistribution of charge caused by the optical intensity. For relatively broad optical beams it can be shown that $E_{\mathrm{sc}}$ is approximately given by [5]

$$
E_{\mathrm{sc}}=E_{0} \frac{I_{\infty}+I_{d}}{I+I_{d}}-\frac{k_{B} T}{e} \frac{\partial I / \partial x}{I+I_{d}},
$$

where the first term relates to charge drift and the latter to charge diffusion. Here $I_{d}$ is the so-called dark irradiance, $I$ $=I(x, z)$ is the power density of the optical beam, which is related to the slowly varying envelope $\phi$ through Poynting's vector, i.e., $I=\left(n_{e} / 2 \eta_{0}\right)|\phi|^{2}$, where $\eta_{0}=\sqrt{\mu_{0} / \epsilon_{0}}$ is the intrinsic impedance of free space, and $I_{\infty}=I(x \rightarrow \pm \infty)$. Furthermore, $k_{B}$ is the Boltzmann's constant, $T$ is the absolute temperature, and $E_{0}$ represents the value of the space-charge field at $x \rightarrow \pm \infty$. If the spatial extent of the optical beam is much less than the $x$-width $W$ of the photorefractive crystal, then $E_{0}$ is approximately $\pm V / W$, where $V$ is the applied external voltage. By substituting Eq. (2) into Eq. (1) and after appropriate normalization we have

$$
i U_{\xi}+\frac{1}{2} U_{s s}+(1+\rho) \frac{U}{1+|U|^{2}}+\gamma \frac{\left(|U|^{2}\right)_{s} U}{1+|U|^{2}}=0,
$$

where $U_{\xi}=\partial U / \partial \xi$, etc. The power density is now normalized with respect to the dark irradiance, i.e., $U=\sqrt{n_{e} /\left(2 \eta_{0} I_{d}\right)} \phi$, the normalized coordinate $\xi$ is related to the actual propagation distance by $\xi=\left(k_{0} n_{e}^{3} r_{33}\left|E_{0}\right| / 2\right) z$ and the dimensionless transverse coordinate is given by $s=\left(k_{0} n_{e}^{2} \sqrt{r_{33}\left|E_{0}\right| / 2}\right) x$. In this equation, $\rho$ stands for the ratio of the optical intensity at the tails of the beam to the dark irradiance, that is $\rho=I_{\infty} / I_{d}$, and $\gamma$ is a parameter associated with diffusion effects that is given by $\gamma=\left(k_{B} T / e\right) k_{0} n_{e}^{2} \sqrt{r_{33} /\left(2\left|E_{0}\right|\right)}$. In deriving Eq. (3), it was assumed that $E_{0}<0$, so that the associated selfdefocusing nonlinearity is capable of supporting the formation of dark and gray solitons.

Under strong bias conditions and for relatively large optical beams, the drift process dominates. In this case, we can neglect the diffusion process, that is, $\gamma=0$, and Eq. (3) takes the form of a nonlinear Schrödinger equation with a higherorder nonlinearity, which exhibits solitary wave solutions of the dark and gray type.

Before analyzing the solitary solutions of the above model, let us apply the following transformation:

$$
U(s, \xi)=A(\eta, \xi) \exp [i \omega(\xi) \eta] \exp [i \alpha(\xi)],
$$

where $\eta$ is a moving coordinate given by $\eta=s-\nu(\xi), \nu(\xi)$ represents the trajectory of the moving transverse coordinate and is such that $\nu^{\prime}(\xi)=\omega(\xi)$, and $\omega(\xi)$, which is associated with the angle between the central wave vector and the propagation axis, plays the role of a transverse velocity. Finally, $\alpha(\xi)$ allows the variation of the phase during propagation in the new coordinate system and satisfies $\alpha^{\prime}(\xi)$ $=\omega^{2}(\xi) / 2$.

The new envelope $A(\eta, \xi)$ satisfies the following evolution equation:

$$
i A_{\xi}+\frac{1}{2} A_{\eta \eta}+(1+\rho) \frac{A}{1+|A|^{2}}+\gamma \frac{\left(|A|^{2}\right)_{\eta} A}{1+|A|^{2}}-\omega^{\prime} \eta A=0 .
$$

Whenever $\omega(\xi)=$ const, say $\omega$, we have $\nu(\xi)=\omega \xi$ and $\alpha(\xi)$ $=\omega^{2} \xi / 2$. In this case, we are in the presence of a Galilean transformation, which showed to be particularly convenient to determine solutions of Eq. (3) with $\gamma=0$, whose phase is constant when $s$ or $\eta \rightarrow \pm \infty$.

These solitary wave solutions are given by $[5,13]$

$$
U(s, \xi)=\sqrt{\rho} y(\eta) \exp \left(i \mu \xi-i J \int_{0}^{\eta} \frac{d \eta^{\prime}}{y^{2}}+i \omega \eta+i \frac{\omega^{2}}{2} \xi+i \theta_{0}\right),
$$

where $\theta_{0}$ is an arbitrary initial phase and $y(\eta)$ is a normalized real function $[0 \leq y(\eta) \leq 1]$ that represents the soliton profile and satisfies the boundary conditions $y(\eta \rightarrow \infty)=1$, $y(0)=\sqrt{m}$, and $y^{\prime}=y^{\prime \prime}=0$ for $\eta \rightarrow \infty$. Note that the parameter $m$ is associated with the minimum intensity of the optical soliton, and also that $m=0$ corresponds to a dark soliton. The normalized field profile obeys the ordinary differential equation

$$
\left(y^{\prime}\right)^{2}-2 \mu\left(y^{2}-1\right)-J^{2}\left(1-\frac{1}{y^{2}}\right)-2 \frac{(1+\rho)}{\rho} \ln \left(\frac{1+\rho}{1+\rho y^{2}}\right)=0,
$$

where $y^{\prime}=d y / d \eta$. Moreover, the phase shift $\mu$ is given by

$$
\mu=\frac{1}{1-m}+\frac{m}{(1-m)^{2}} \frac{(1+\rho)}{\rho} \ln \left(\frac{1+\rho m}{1+\rho}\right),
$$

and the constant $J$ satisfies $J^{2}=2(1-\mu)$. The condition for constant phase at infinity implies that $\omega=J$, hence the transverse velocity depends on the solitary wave characteristics through the expression

$$
\omega^{2}=\frac{2 m}{1-m}\left[\frac{1}{1-m} \frac{1+\rho}{\rho} \ln \left(\frac{1+\rho}{1+\rho m}\right)-1\right],
$$

and, furthermore, $\omega$ can be a positive or negative quantity as it happens with $J$. In the case of dark solitons, we have $m$ $=0$, which results in a null transverse velocity.

At this point, it should be mentioned that Eq. (7) does not allow closed-form solutions and, therefore, the envelope profile must be obtained numerically by integrating this equation.

\section{DIFFUSION EFFECTS}

Let us now consider the effects of the diffusion process in the propagation of dark and gray solitons. These effects can be directly observed by numerically solving Eq. (3) using a beam propagation method, with the solitary waves (obtained above for $\gamma=0$ ) as the input beams. As observed for bright solitons in the photorefractive focusing model [14-17], the trajectories of dark and gray beams are curvilinear [Fig. 1(a)]. Moreover, our simulation results also show that the shape of the optical beam remains approximately constant 


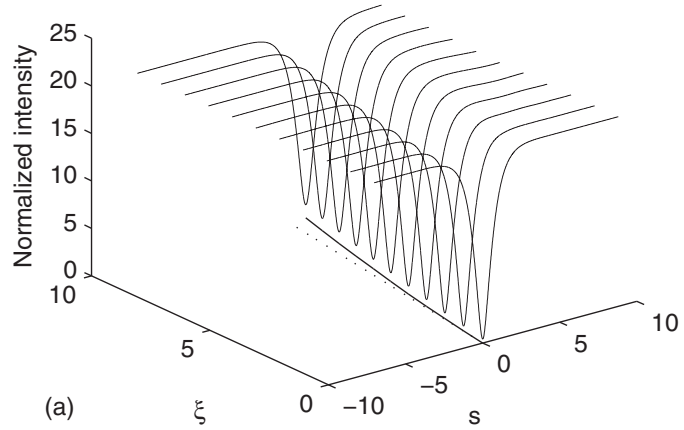

Keeping in mind the numerical simulation results, we assume that the solution of Eq. (3) in the presence of diffusion effects is again given by Eqs. (6)-(9), but now with $m$ being dependent on the propagation distance $\xi$. As a consequence, the beam profile $y$, the phase shift $\mu$, and the transverse velocity $\omega$ are also functions of the propagation distance. In turn, this implies that the trajectory $\eta=s-\nu(\xi)$, with $\nu^{\prime}(\xi)$ $=\omega(\xi)$, will be curvilinear, as observed by numerical simulations. The dependence of the wave characteristics on the propagation distance can then be obtained by substituting Eq. (6) into Eq. (11), which yields

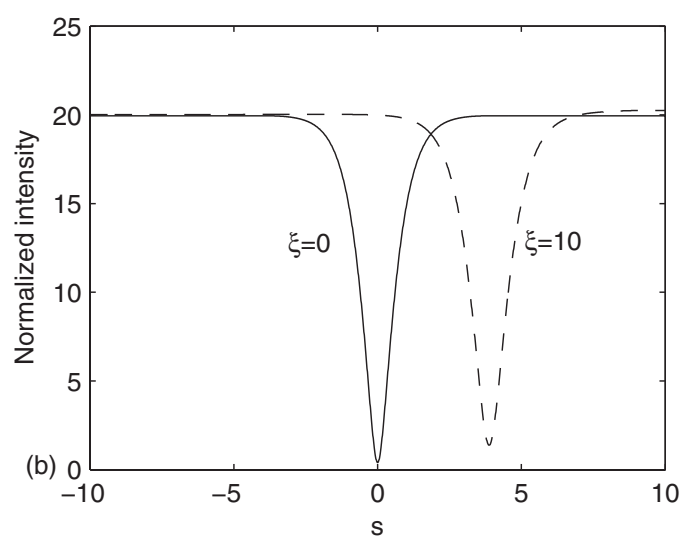

$$
\frac{d}{d \xi}\left[\omega \int_{-\infty}^{\infty} d \eta\left(y-\frac{1}{y}\right)^{2}\right]=-4 \gamma \rho \int_{-\infty}^{\infty} d \eta \frac{y^{2}\left(y^{\prime}\right)^{2}}{1+\rho y^{2}} .
$$

Since the envelope profile $y(\eta)$ depends on $m$, the two integrals present in this equation will be functions of $m$, and, thus, also functions of $\xi$. Moreover, given that $m$ and $\omega$ are related through Eq. (9), we can write the previous equation in the form

$$
\frac{d \omega}{d \xi}=-4 \gamma \rho \frac{\int_{-\infty}^{\infty} d \eta \frac{y^{2}\left(y^{\prime}\right)^{2}}{1+\rho y^{2}}}{\frac{d}{d \omega}\left[\omega \int_{-\infty}^{\infty} d \eta\left(y-\frac{1}{y}\right)^{2}\right]} .
$$

FIG. 1. (a) Evolution of the normalized intensity of a gray soliton and beam trajectory $(m=0.02, \rho=20$, and $\gamma=0.01)$; the dotted line represents the beam trajectory in the absence of diffusion. (b) Intensity profiles at $\xi=0$ and $\xi=10$.

during propagation. Nonetheless, the minimum value attained by the profile varies considerably, as illustrated in Fig. 1(b). This is in clear contrast with the bright soliton case, where the maximum intensity value remained approximately constant [15].

These self-bending effects can be systematically studied using a perturbation method that makes use of the conservation laws associated to Eq. (3). More specifically, we consider the conservation of the system momentum which is obtained by multiplying Eq. (3) with $U_{s}^{*}$, and then adding the result with its complex conjugate. Bearing in mind that we are considering optical beams with nonzero tails, this conservation statement must be renormalized and, in the absence of diffusion effects, it is given by $[21,22]$

$$
\frac{d}{d \xi}\left(\frac{i}{2} \int_{-\infty}^{\infty} d s\left(U U_{s}^{*}-U^{*} U_{s}\right)-\left.\rho \operatorname{Arg} U\right|_{s=-\infty} ^{s=+\infty}\right)=0,
$$

where $\operatorname{Arg} U$ represents the phase of $U$. It is interesting to note that, as a result of the moving coordinate system considered, the system momentum is a finite quantity. When diffusion effects are taken into account in Eq. (3), the previous conservation law takes the modified form

$$
\begin{aligned}
& \frac{d}{d \xi}\left[\frac{i}{2} \int_{-\infty}^{\infty} d s\left(U U_{s}^{*}-U^{*} U_{s}\right)\left(1-\frac{\rho}{|U|^{2}}\right)\right] \\
& \quad=-\gamma \int_{-\infty}^{\infty} d s \frac{\left[\left(|U|^{2}\right)_{s}\right]^{2}}{1+|U|^{2}},
\end{aligned}
$$

where we have also used $\operatorname{Arg} U=(i / 2) \ln \left(U^{*} / U\right)$.

Note that the right-hand side of this equation is a function of $\omega$ and, therefore, we can think of Eq. (13) as a differential equation which allows the evaluation of $\omega(\xi)$. Unfortunately, the nonexistence of a closed-form solution for the envelope profile $y(\eta)$ requires that the two integrals present in this equation be evaluated using numerical procedures, which prevent us from obtaining a general analytical expression for $\omega(\xi)$. Nevertheless, this equation can always be numerically integrated. Furthermore, an approximate expression for the soliton velocity can be easily obtained by considering the first terms of its Taylor series expansion in the vicinity of $\xi$ $=0$. To first order, we have

$$
\omega(\xi) \cong \omega_{0}+\gamma K \xi
$$

where $\omega_{0}=\omega(\xi=0)$ and the product $\gamma K$ is simply the righthand side of Eq. (13) evaluated at $\xi=0$, that is, $\gamma K=\omega^{\prime}(0)$. The function $K$ represents a self-bending coefficient and depends on parameters $\rho$ and $m$, more specifically, on $\rho$ and $m_{0}=m(\xi=0)$. It is important to point out that this function does not depend on the sign of the initial transverse velocity $\omega_{0}$. Effectively, since the two integrals present in the definition of $\omega^{\prime}$ are even functions of $\omega$ (they depend on $m$, which in turn is related to $\omega^{2}$ ) we can conclude that $\omega^{\prime}$ and $K$ are both even functions of $\omega$. Moreover, it is also important to mention that the denominator in Eq. (13) represents $(d P / d \omega) / \rho$, where $P$ is the renormalized system momentum. The sign of $d P / d \omega$ is known to be associated with the stability of dark and gray solitons $[13,24]$. More specifically, these beams are stable when $d P / d \omega<0$, and unstable otherwise. Note that, in photorefractive media and in the absence of diffusion, the instability region corresponds to solitons with approximately $\rho \geq 40$ and very small values of $m_{0}$ [13]. 

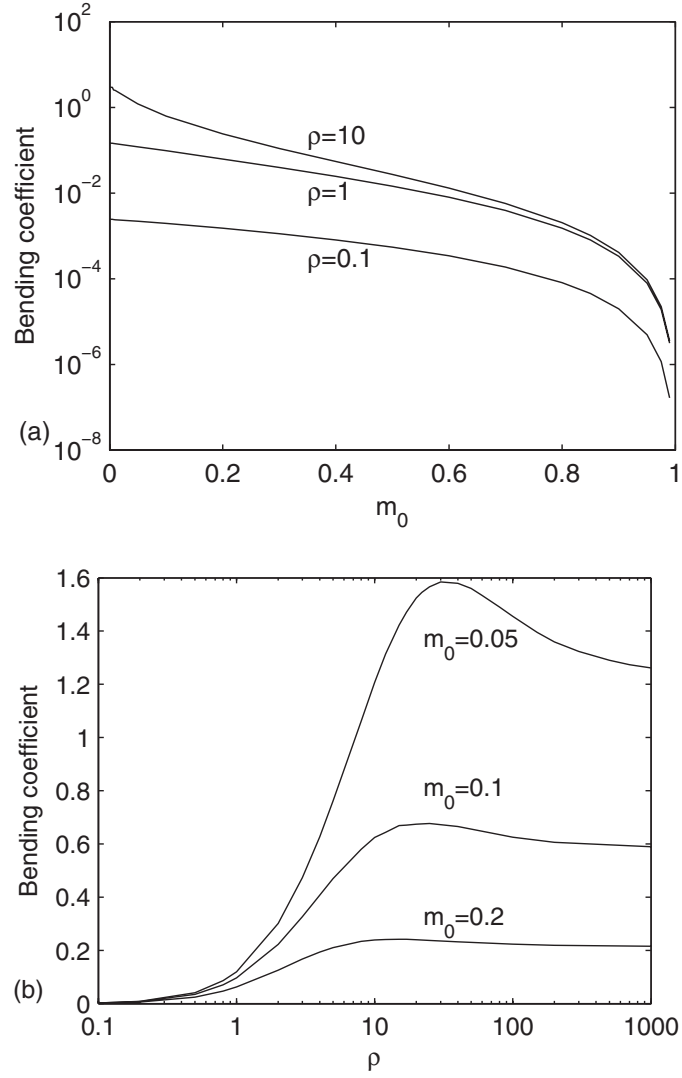

FIG. 2. Dependence of the self-bending coefficient $K$ on (a) parameter $m_{0}$ for $\rho=0.1,1$, and 10 ; (b) parameter $\rho$ for $m_{0}=0.05$, 0.1 , and 0.2 .

In the following analysis, we will consider only stable solitons, that is, beams with initial profiles lying outside this instability region [25]. For these solitons, it is straightforward to show from Eq. (13) that the self-bending coefficient is always positive. Since $\gamma>0$, then $\omega^{\prime}(0)$ is positive too and, therefore, the absolute value of the soliton transverse velocity can initially increase or decrease depending on the sign of $\omega_{0}$.

The value of the self-bending coefficient can be numerically evaluated, using the solutions of Eq. (7) for a given set of parameters $\rho$ and $m_{0}$. The derivative appearing in the denominator of Eq. (13) has been computed using a finite difference approach. Figure 2 shows the dependence of function $K$ on parameters $\rho$ and $m_{0}$ for some soliton parameters. Even though the variation of $K$ with $m_{0}$ is only depicted for three values of $\rho$, our calculations show that the self-bending coefficient of stable solitons is a decreasing function of $m_{0}$. The dependence on parameter $\rho$ is slightly more complicated. The self-bending coefficient exhibits a maximum for a value of $\rho$ that depends on $m_{0}$, and then approaches a saturation value for large $\rho$. Naturally, both the maximum $K$ and its saturation value depend on $m_{0}$, being decreasing functions of this parameter. This behavior of the self-bending coefficient is well understood if we realize that the width of the normalized beam increases with $m_{0}$ and, furthermore, has a minimum for $\rho$ around the same value for which there is a peak in Fig. 2(b) [13]. Hence, as expected, broader beams suffer less self-bending.
Having determined an approximate expression of the transverse velocity $\omega(\xi)$, it is now possible to obtain the variation of the minimum optical intensity with $\xi$, as well as the trajectory of the optical beam under diffusion effects. Assuming that in the moving coordinate the beam profile minimum is at $\eta_{\min }=0$ then the beam trajectory is given by $s_{\min }=\nu(\xi)$, where $\nu^{\prime}=\omega$. Using Eq. (14), to first order and in the neighborhood of $\xi=0$, we have

$$
s_{\min } \cong \omega_{0} \xi+\frac{1}{2} \gamma K \xi^{2}
$$

where it was assumed that $\nu(0)=0$. This equation clearly shows that, as a result of the diffusion process, the beam trajectory is deflected, and the beam center moves along a parabolic line. The deviation from the initial trajectory is given by $\Delta s_{\min }=s_{\min }-\omega_{0} \xi=\gamma K \xi^{2} / 2$, and depends not only on the diffusion parameter, but also on the soliton characteristics through the self-bending coefficient. In actual coordinates, this deviation takes the form $\Delta x_{\min }=\left(k_{B} T / 8 e\right)$ $\times\left(k_{0} n_{e}^{3} r_{33}\right)^{2}\left|E_{0}\right| K z^{2}$, which indicates that the shift of the trajectory of dark and gray stable solitons is always in the positive $x$ direction. While the previous result could indicate that the degree of bending increases linearly with the applied field (which is not consistent with an expected drift dominance for high applied fields), we should stress that, for higher $E_{0}$, a given $K$ ( $\rho$ and $m_{0}$ fixed) corresponds to a narrower real beam. For a better understanding of the actual importance of $E_{0}$ and the actual width of the beam on the diffusion effects, we have fixed $\rho$ and allowed $m_{0}$ to vary in order to obtain beams of equal full width at half maximum $x_{\text {fwhm }}$ under different external applied field $E_{0}$, or to obtain beams of different $x_{\text {fwhm }}$ under the same applied field $E_{0}$ (Fig. $3)$. As expected, the self-bending is negligible for higher values of $E_{0}$, since the diffusion transport mechanism can be neglected when compared to the drift counterpart. Also, broader beams suffer less self-bending since diffusion is a nonlocal mechanism that depends on the strength of the spatial derivative of the intensity profile. At this point, it is noteworthy pointing out that these results are similar to the ones obtained for bright solitons in biased photorefractive media [15]. However, there is a significant difference concerning the bending direction, since bright solitary beams bend toward the negative $x$ direction. Keeping in mind that the sign of $E_{0}$ is associated with the type of solitons, that is, $E_{0}>0$ for bright solitons, and $E_{0}<0$ for dark and gray ones, we can then conclude that the trajectories of optical screening solitons in photorefractive material always bend in the direction opposite to the applied field.

In turn, the variation of $m$ with the propagation distance can be approximately obtained with the help of Eqs. (9) and (14), and using a Taylor series expansion. Once again to first order and in the vicinity of $\xi=0$ we have

$$
m(\xi) \cong m_{0}+m^{\prime}(0) z
$$

where $m^{\prime}(0)$ can be obtained from Eq. (9) as $m^{\prime}(0)$ $=\left.\left[2 \omega \omega^{\prime} /\left(d \omega^{2} / d m\right)\right]\right|_{\xi=0}$, or 

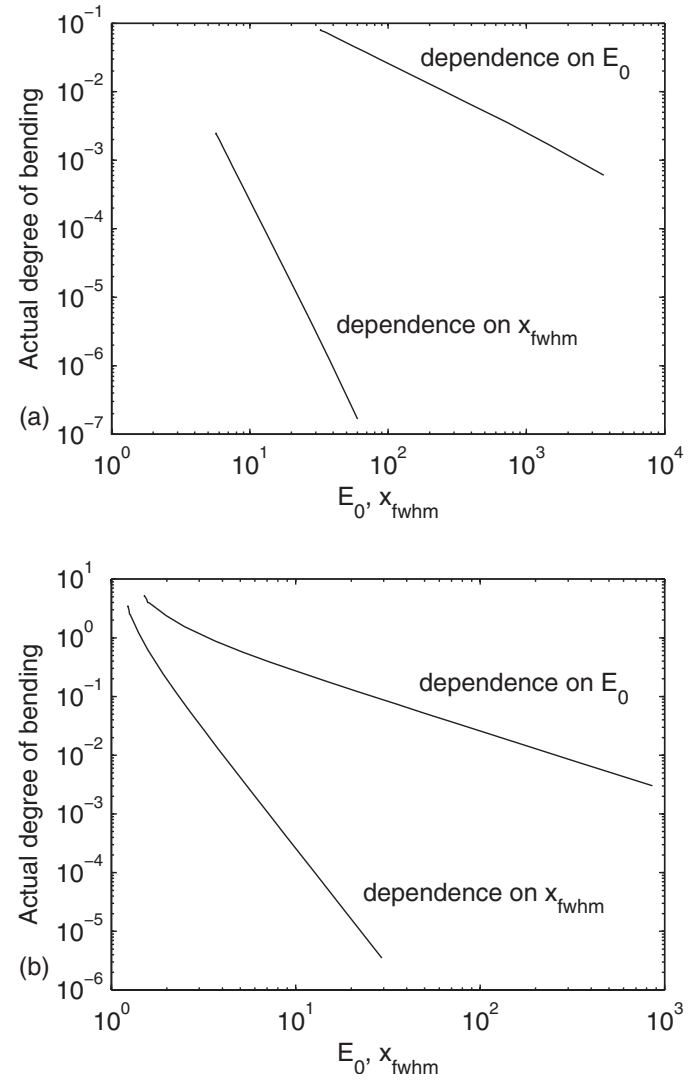

FIG. 3. Dependence of the actual degree of bending, given by $\left(k_{B} T / 4 e\right)\left(k_{0} n_{e}^{3} r_{33}\right)^{2}\left|E_{0}\right| K$, on the applied electric field $E_{0}$ and on the full width at half maximum $x_{\text {fwhm }}$ for (a) $\rho=0.1$ and (b) $\rho=10$. The represented degree of bending, $E_{0}$ and $x_{\text {fwhm }}$ are normalized by $k_{B} \operatorname{Tr}_{33}\left(k_{0} n_{e}^{3}\right)^{2} /(4 e), r_{33}^{-1}$ and $\sqrt{2} /\left(k_{0} n_{e}^{2}\right)$, respectively. Without loss of generality and whenever fixed $E_{0}$ is taken to be $r_{33}^{-1}$ and $x_{\mathrm{fwhm}}$ is taken to be $\sqrt{2} /\left(k_{0} n_{e}^{2}\right)$. Note that the minimum value of the $x$ range in both graphs is imposed by the existence of solitons.

$$
m^{\prime}(0)=\frac{\omega_{0}\left(1-m_{0}\right)^{2} \omega^{\prime}(0)}{\frac{1+m_{0}}{1-m_{0}} \frac{1+\rho}{\rho} \ln \left(\frac{1+\rho}{1+\rho m_{0}}\right)-\frac{m_{0}(1+\rho)}{1+\rho m_{0}}-1}
$$

It can be shown that the denominator on the right-hand side of Eq. (17), which is associated with $\left.\left(d \omega^{2} / d m\right)\right|_{\xi=0}$, is always greater than zero. Moreover, taking into account that $\omega^{\prime}(0)$ is positive, this implies that $m^{\prime}(0)$ has the same sign as the initial transverse velocity $\omega_{0}$. Hence, for the region of validity of the previous equation, positive (negative) initial velocities will be associated with an increase (decrease) in the value of the minimum.

To illustrate our results, let us consider the evolution of gray solitons, when the diffusion parameter is $\gamma=0.01$ and for a propagation distance of $\xi=10$. Figure 4 compares the deviation of the beam center from its initial trajectory and the variation of the beam minimum intensity predicted by our model with those found by numerically solving Eq. (3). In this figure, different values of $\rho$ and $m_{0}$, and both positive and negative initial velocities, have been considered. Also
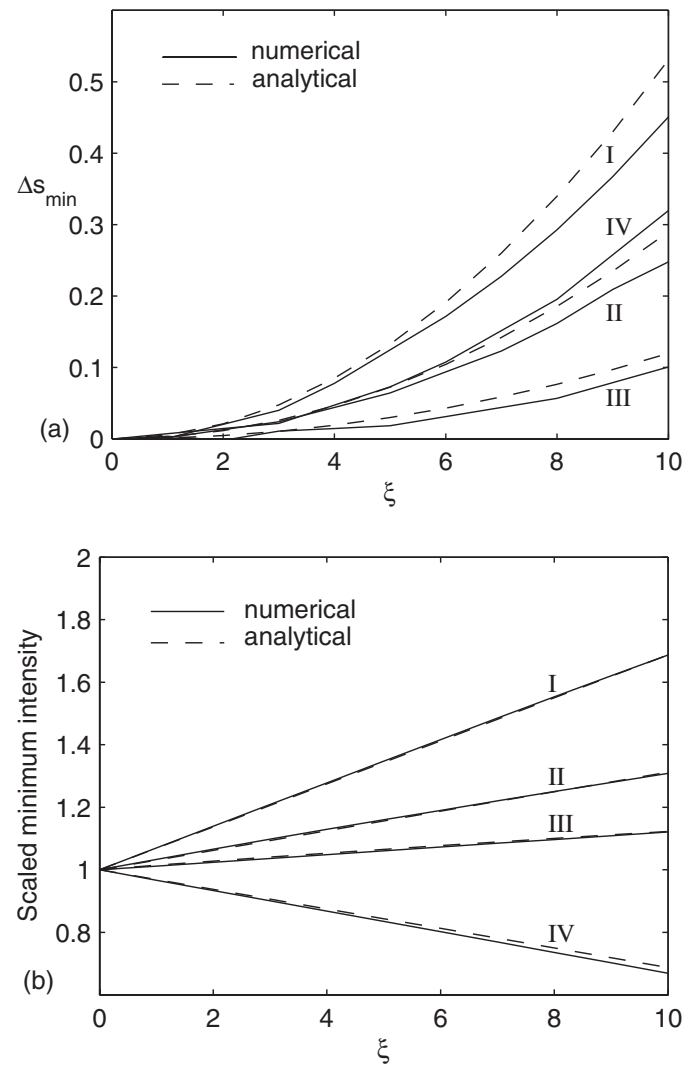

FIG. 4. Comparison of numerical and analytical results when $\gamma=0.01$. (a) Trajectory of the beam center; (b) scaled minimum intensity. Case I: $\rho=8, m_{0}=0.05$, and $\omega_{0}>0$. Case II: $\rho=8, m_{0}$ $=0.1$, and $\omega_{0}>0$. Case III: $\rho=20, m_{0}=0.2$, and $\omega_{0}>0$. Case IV: $\rho=8, m_{0}=0.1$, and $\omega_{0}<0$.

note that in order to facilitate the comparison between the different cases represented, the variation of the minimum intensity is illustrated by the evolution of $m(\xi) / m(0)$. As one can see, the analytical approach is generally in good agreement with the numerical results. By comparing the evolution of solitons I-III, which correspond to $m_{0}=0.05,0.1$, and 0.2 , respectively, it is possible to verify that diffusion effects are more notorious for small values of $m_{0}$. It is also interesting to compare the evolution of the gray solitons represented in cases II and IV, which differ only in the sign of the initial transverse velocity. As expected, the minimum intensity increases when $\omega_{0}>0$, decreasing otherwise. Moreover, the approximate expression derived for $\Delta s_{\min }$ predicts a selfbending independent of the sign of $\omega_{0}$. However, it can be verified in Fig. 4(a) that the numerical results obtained for cases II and IV are slightly different, thus indicating that the higher order terms not considered in Eq. (14) depend on the sign of $\omega_{0}$.

It is also important to note that the application of Eq. (16) to the case of dark solitons will give erroneous results. In effect, since the initial transverse velocity $\omega_{0}$ of these beams is zero, Eq. (17) predicts that $m^{\prime}(0)=0$, which implies a constant $m$ for dark solitons. Nonetheless, the results of our numerical simulations showed that although this variation is not considerable, it still can be observed. Therefore, a better approximation of $m(\xi)$ is required for dark solitons. In the 

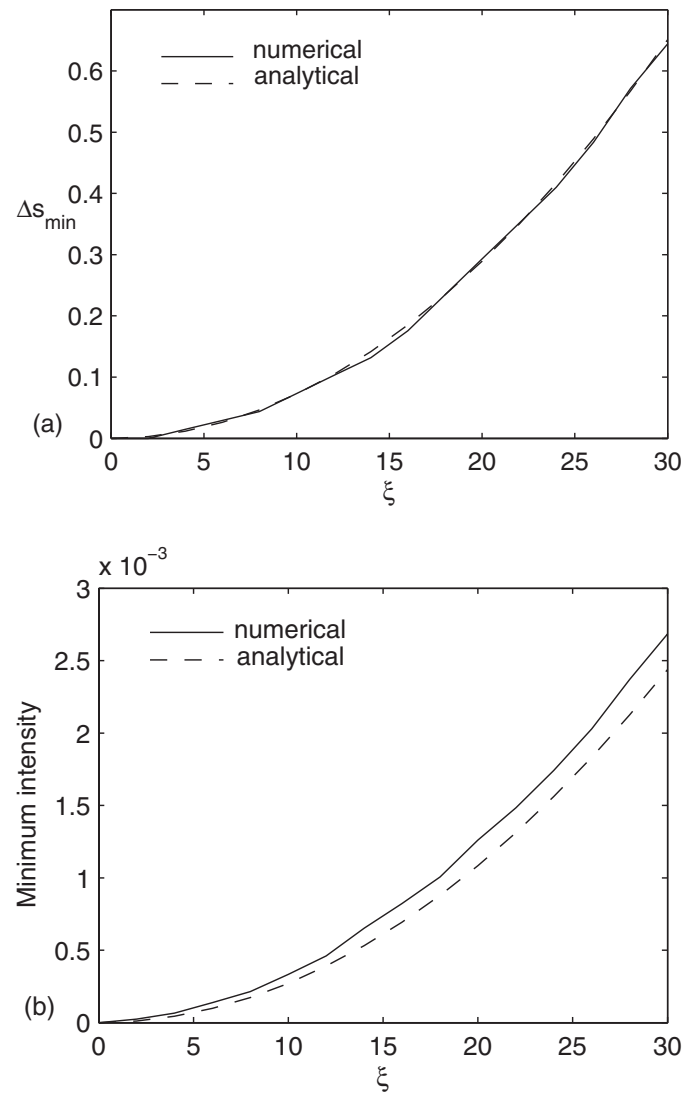

FIG. 5. Comparison of numerical and analytical results for a dark soliton $\left(m_{0}=0\right)$ with $\rho=40$, when $\gamma=0.01$. (a) Position of beam center; (b) minimum intensity.

vicinity of $\xi=0$ and to second order in $\xi$, we obtain for the case of dark solitons $m \cong m^{\prime \prime}(0) \xi^{2} / 2$, where

$$
m^{\prime \prime}(0)=\frac{\omega^{\prime}(0)^{2}}{\frac{1+\rho}{\rho} \ln (1+\rho)-1} .
$$

Figure 5 presents the comparison between the numerical and analytical results for a dark soliton with $\rho=40$ and $\gamma$ $=0.01$, for a propagation distance of $\xi=30$. In particular, the evolution of the normalized minimum optical intensity, illustrated in Fig. 5(b), shows that the second-order expansion to $m(\xi)$ is in good agreement with the numerical results.

The evolution of both the trajectory and the minimum intensity of a gray soliton with a small minimum intensity $\left(m_{0}=0.01\right)$ and a negative initial velocity is illustrated in Fig. 6. Our numerical simulations indicate that the absolute value of the transverse velocity $\omega$ will decrease, until it becomes zero. Similarly, as predicted by Eq. (9), the minimum intensity of the optical beam decreases, and when $\omega=0$ we also have $m=0$. This means that the gray soliton becomes a dark soliton. From that point on, both the transverse velocity and the minimum intensity increase, and the dark soliton again becomes a gray soliton. As shown in Fig. 6(a), the analytical results for the trajectory of this optical beam agree well with the numerical simulations. On the contrary, the first-order analytical results for the variation of the minimum, given by
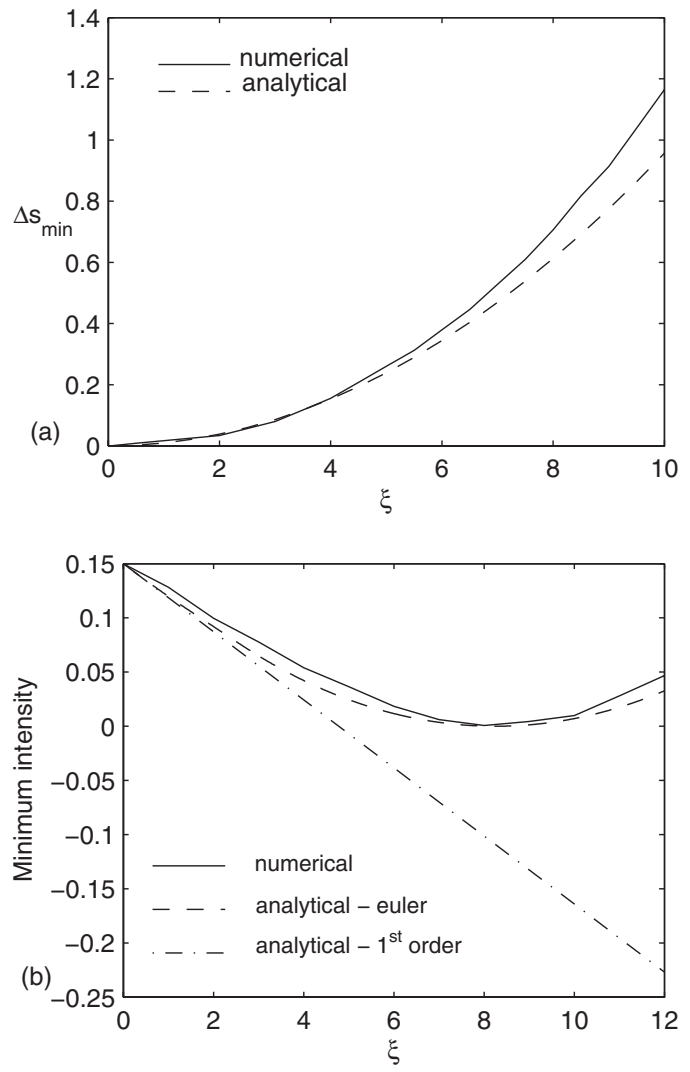

FIG. 6. Comparison of numerical and analytical results for a gray soliton with $m_{0}=0.01$ and $\rho=15$, when $\gamma=0.005$ and $\omega_{0}<0$. (a) Position of beam center; (b) minimum intensity.

Eq. (16), are not very good. In effect, they are obtained from an expansion around $\xi=0$, and therefore do not take into account the change in the sign of $\omega$. Nevertheless, these analytical results can be improved by numerically solving the differential equation for $\omega^{\prime}(\xi)$ [Eq. (13)]. This can be easily achieved by simply dividing the propagation distance in smaller steps, and by adjusting the self-bending coefficient at each step, which corresponds to integrate the differential equation by Euler's method. The results obtained by this method, also depicted in Fig. 6(b), are in good agreement with the numerical simulations. Furthermore, it should be referred that our numerical simulations indicate that the evolution of the beam trajectory and its minimum intensity for larger propagation distances can also be predicted by numerical integrating Eq. (13).

The low-amplitude case also deserves special attention. In this limit, that is, when $\rho \ll 1$ or $|U|^{2} \ll 1$, Eq. (3) takes the form

$$
i U_{\xi}+\frac{1}{2} U_{s s}+(1+\rho) U-|U|^{2} U+\gamma\left(|U|^{2}\right)_{s} U=0,
$$

which is a modified version of the nonlinear Schrödinger equation. It is interesting to point out that this equation is analogous to the equation describing the evolution of temporal pulses under the influence of intrapulse Raman scattering, which is known to be associated with the phenomenon of soliton self-frequency shift already studied [20-24]. 
Unlike Eq. (3), the previous evolution equation (with $\gamma$ $=0$ ) exhibits dark and gray solitons which are given in an analytical form [26]. The action of diffusion on the behavior of these Kerr-type solitons can then be investigated by simply substituting the low amplitude beam profile in Eq. (13), in which case we obtain $d \omega / d \xi=4 \gamma\left(\rho-\omega^{2}\right)^{2} / 15$. Even though this ordinary differential equation can be easily integrated, it is not possible to solve it for $\omega$ in a closed form. However, as before, an approximate expression for the soliton velocity $\omega(\xi)$ can be obtained by considering the firstorder term of its Taylor series expansion in the vicinity of $\xi=0$,

$$
\omega(\xi) \cong \omega_{0}+\frac{4}{15} \gamma \rho^{2}\left(1-m_{0}\right)^{2} \xi .
$$

It is noteworthy pointing out that the low amplitude selfbending coefficient is given by $K=4 \rho^{2}\left(1-m_{0}\right)^{2} / 15$, which is in excellent agreement with the numerical value obtained for the general case when $\rho \ll 1$. Furthermore, in the case of a dark soliton, $m_{0}=0$ and $\omega_{0}=0$, and so the previous equation reduces to $\omega(\xi) \cong 4 \gamma \rho^{2} \xi / 15$. Analogously to the general case presented earlier, the trajectory of a low amplitude optical beam and its minimum intensity evolution can now be approximately determined from Eq. (20), and are given by $s_{\min } \cong \omega_{0} \xi+\frac{2}{15} \gamma \rho^{2}\left(1-m_{0}\right)^{2} \xi^{2}$ and $m(\xi) \cong m_{0}+\frac{8}{15} \beta \gamma \rho \sqrt{\rho m_{0}}(1$ $\left.-m_{0}\right)^{2} \xi$, where once again it was assumed that $\nu(0)=0$. Similarly to the general case, a second-order approximation of $m(\xi)$ is required for dark solitons, yielding $m(\xi)$ $\cong 16 \gamma^{2} \rho^{3} \xi^{2} /(15)^{2}$ for this type of optical beams. In actual coordinates, the deviation from the initial trajectory in the low-amplitude regime can be easily obtained as $\Delta x_{\text {min }}$ $=\left[K_{B} T /(30 e)\right]\left(k_{0} n_{e}^{3} r_{33}\right)^{2}\left|E_{0}\right| \rho^{2}\left(1-m_{0}\right)^{2} z^{2}$ or, equivalently, as a function of $E_{0}$ and $x_{\mathrm{fwhm}}, \quad \Delta x_{\min }=\left[K_{B} T /(30 e)\right]$ $\times\left[38.6 /\left(k_{0}^{2} n_{e}^{2}\left|E_{0}\right| x_{\text {fwhm }}^{4}\right)\right] z^{2}$. It is interesting to compare this deviation with the one predicted for bright solitons, which is given by [15] $\Delta x_{\mathrm{MAX}}=-\left[K_{B} T /(15 e)\right]\left(k_{0} n_{e}^{3} r_{33}\right)^{2} E_{0} r^{2} z^{2}$, where $r$ is the ratio of the soliton maximum intensity to the dark irradiance, or $\Delta x_{\mathrm{MAX}}=-\left[K_{B} T /(15 e)\right]\left[38.6 /\left(k_{0}^{2} n_{e}^{2} E_{0} x_{\mathrm{fwhm}}^{4}\right)\right] z^{2}$. In addition to the expected dependence on soliton parameters, these two deviations also differ in their signs. Furthermore, the previous expressions also show that the bending of bright solitons is twice the one suffered by dark or gray ones with the same spatial width.

\section{CONCLUSIONS}

The effects of the diffusion process on the evolution of dark and gray photorefractive solitons have been systematically investigated. By employing numerical techniques we have found that these optical beams experience a modification of their initial trajectory, as well as a variation of their minimum intensity. This process was further studied using a perturbation method which involves the modified conservation laws of the nonlinear wave equation in a moving coordinate system, yielding a differential equation that governs the evolution of the transverse velocity of the optical beam. Our analysis indicates that the deviation of the center of the solitary beam from its initial trajectory is approximately parabolic, whereas the minimum intensity increases or decreases linearly with the propagation distance, depending on the sign of its initial transverse velocity. Moreover, the dependence of these diffusion effects on the value of the external bias electric field and on soliton parameters was also considered in detail. The analytical results were then compared to those obtained numerically and were found to be in good agreement with each other.
[1] G. C. Duree, J. L. Shultz, G. J. Salamo, M. Segev, A. Yariv, B. Crosignani, P. DiPorto, E. J. Sharp, and R. R. Neurgaonkar, Phys. Rev. Lett. 71, 533 (1993).

[2] G. C. Duree, G. J. Salamo, M. Segev, A. Yariv, B. Crosignani, P. D. Porto, and E. J. Sharp, Opt. Lett. 19, 1195 (1994).

[3] M. Shih, M. Segev, G. C. Valley, G. Salamo, B. Crosignani, and P. DiPorto, Electron. Lett. 31, 826 (1995).

[4] M. Segev, G. C. Valley, B. Crosignani, P. DiPorto, and A. Yariv, Phys. Rev. Lett. 73, 3211 (1994).

[5] D. N. Christodoulides and M. I. Carvalho, J. Opt. Soc. Am. B 12, 1628 (1995).

[6] M. Segev, G. C. Valley, S. R. Singh, M. I. Carvalho, and D. N. Christodoulides, Opt. Lett. 20, 1764 (1995).

[7] M. I. Carvalho, S. R. Singh, D. N. Christodoulides, and R. I. Joseph, Phys. Rev. E 53, R53 (1996).

[8] D. N. Christodoulides, S. R. Singh, M. I. Carvalho, and M. Segev, Appl. Phys. Lett. 68, 1763 (1996).

[9] D. N. Christodoulides, T. H. Coskun, M. Mitchell, and M. Segev, Phys. Rev. Lett. 78, 646 (1997).

[10] M. Mitchell and M. Segev, Nature (London) 387, 880 (1997).

[11] N. K. Efremidis, S. Sears, D. N. Christodoulides, J. W. Fleischer, and M. Segev, Phys. Rev. E 66, 046602 (2002).
[12] J. W. Fleischer, M. Segev, N. K. Efremidis, and D. N. Christodoulides, Nature (London) 422, 147 (2003).

[13] A. G. Grandpierre, D. N. Christodoulides, T. Coskun, M. Segev, and Y. Kivshar, J. Opt. Soc. Am. B 18, 55 (2001).

[14] M. Shih, P. Leach, M. Segev, G. Salamo, M. H. Garret, and G. C. Valley, Opt. Lett. 21, 324 (1996).

[15] M. I. Carvalho, S. R. Singh, and D. N. Christodoulides, Opt. Commun. 120, 311 (1995).

[16] L. Jinsong and L. Keqing, J. Opt. Soc. Am. B 16, 550 (1999).

[17] V. Aleshkevich, Y. Kartashov, and V. Vysloukh, Phys. Rev. E 63, 016603 (2000).

[18] D. F. Parker, C. Sophocleous, and C. Radha, J. Phys. A 35, 1283 (2002).

[19] M. Facão and D. F. Parker, Phys. Rev. E 68, 016610 (2003).

[20] Y. Kivshar and V. V. Afanasjev, Opt. Lett. 16, 285 (1991).

[21] I. M. Uzunov and V. S. Gerdjikov, Phys. Rev. A 47, 1582 (1993).

[22] Y. S. Kivshar and X. Yang, Phys. Rev. E 49, 1657 (1994).

[23] X. Chen and Z. Chen, J. Opt. Soc. Am. B 15, 2738 (1998).

[24] Y. S. Kivshar and B. Luther-Davies, Phys. Rep. 298, 81 (1998). 
[25] Our numerical simulations have shown that the diffusion process does not drastically change the stability of initially stable dark and gray solitons.

[26] In the absence of diffusion, the dark and gray soliton solu- tions of Eq. (19) are given by $U(s, \xi)=\sqrt{\rho} y(\eta)$ $\times \exp \left\{-i \beta \tan ^{-1}\left[a \tanh (\sqrt{\rho} a \eta) / \sqrt{1-a^{2}}\right]\right\} \exp \left[i\left(\xi+\theta_{0}\right)\right]$, where $y(\eta)=\sqrt{1-a^{2} \operatorname{sech}^{2}(\sqrt{\rho} a \eta)}, a=\sqrt{1-m}$, and $\beta=\omega /|\omega|$, with $\omega^{2}$ $=\rho m$ in order to prevent infinite phase values in the tails. 